Comparison of electrical and vibration analysis methods for mechanical fault monitoring in wind turbine drivetrains

\begin{tabular}{|r|l|}
\hline Journal: & CM 2018 and MFPT 2018 \\
\hline Manuscript ID & CM-MFPT-0031-2018.R1 \\
\hline Topic: & Machinery diagnostics \\
\hline Date Submitted by the Author: & n/a \\
\hline Complete List of Authors: & Granjon, Pierre; Grenoble University, gipsa-lab \\
\hline Keywords: & Condition monitoring, three-component signal processing \\
\hline
\end{tabular}




\section{Comparison of electrical and vibration analysis methods for mechanical fault monitoring in wind turbine drivetrains \\ Pierre Granjon, Pedro Dias Longhitano and Arshpreet Sing \\ Univ. Grenoble Alpes, CNRS, Grenoble INP*, GIPSA-lab, 38000 Grenoble, France \\ * Institute of Engineering Univ. Grenoble Alpes}

Mechanical faults occurring in drivetrains are traditionally monitored through vibration analysis, and more rarely by analyzing electrical quantities measured on the involved electromechanical system. However, a monitoring method able to take into account the whole information contained in three-phase electrical quantities was recently proposed. The goal of this paper is to compare this three-phase electrical approach and the usual vibration-based method in terms of detection capabilities of mechanical faults in drivetrains.

In this context, a $2 \mathrm{MW}$ geared wind turbine operating in an industrial wind farm was equipped during several months with accelerometers near the main bearing and electrical sensors on the stator of the electrical generator. During this period, an important mechanical fault occurred in the main bearing of this system. The evolution of the fault indicators computed by the two previous approaches are compared all along this period of time. All the indicators behave similarly and show the development of an inner bearing fault in the main bearing.

This demonstrate that a mechanical fault occurring in a drive train can be monitored and detected by analyzing electrical quantities, even if the fault is distant from the electrical generator. 


\title{
Comparison of electrical and vibration analysis methods for mechanical fault monitoring in wind turbine drive trains
}

\author{
Pierre Granjon, Pedro Dias Lonhitano and Arshpreet Singh \\ Univ. Grenoble Alpes, Grenoble INP*, GIPSA-lab, 38000 Grenoble, France \\ * Institute of Engineering Univ. Grenoble Alpes \\ pierre.granjon@grenoble-inp.fr
}

\begin{abstract}
Mechanical faults occurring in drive trains are traditionally monitored through vibration analysis, and more rarely by analyzing electrical quantities measured on the involved electromechanical system. However, a monitoring method able to take into account the whole information contained in three-phase electrical quantities was recently proposed. The goal of this paper is to compare this three-phase electrical approach and the usual vibration-based method in terms of detection capabilities of mechanical faults in drive trains. In this context, a $2 \mathrm{MW}$ geared wind turbine operating in an industrial wind farm was equipped during several months with accelerometers near the main bearing and electrical sensors on the stator of the electrical generator. During this period, an important mechanical fault occurred in the main bearing of this system. The evolution of the fault indicators computed by the two previous approaches are compared all along this period of time. All the indicators behave similarly and show the development of an inner bearing fault in the main bearing. This demonstrate that a mechanical fault occurring in a drive train can be monitored and detected by analyzing electrical quantities, even if the fault is distant from the electrical generator.
\end{abstract}

\section{Introduction}

Onshore or offshore wind turbines are still a developing technology. Though their reliability has improved in time, it can be further increased by implementing efficient condition monitoring systems and predictive maintenance strategies. Currently, vibration analysis seems to be the most popular condition monitoring technique for the mechanical parts of their drive train, such as gears or bearings. However, other possibilities exist, such as analyzing the electrical quantities generated by the output electrical generator driven by the drive train. For example, a method has been recently proposed in [7] to detect mechanical faults in electrical rotating machines using the whole information contained in three-phase electrical currents. Such an electrical approach led to very encouraging results when applied on a testbench emulating a wind turbine. Consequently, the goal of this paper is to apply the same strategy on an industrial wind turbine and check if in this case the same positive results can be obtained. For this, the next section of this paper describes the monitored wind turbine and the available database, while the fault to be detected and its vibratory and electrical signatures are detailed in section 3. Section 4 explains the data selection process applied to the whole database, and the results obtained by using vibration and electrical data are described and compared in section 5 and 6. Finally, the last section of this paper presents the overall conclusions of this research work. 


\section{Process and measurements}

The process of interest is an industrial onshore wind turbine installed in the wind farm of Arfons-Sor located in France (see Fig. 1) and owned by Valemo. This wind farm is constituted by eleven wind turbines of type Eco 802.0 from ALSTOM Ecotecnia (see Fig. 2.a) with the same characteristics: $2 \mathrm{MW}$ rated power, $80 \mathrm{~m}$ rotor diameter, $70 \mathrm{~m}$ hub height. The main components of their drive train are given in Fig. 2.b, and consist in a main bearing supporting the low-speed shaft driven by the hub, a planetary gearbox followed by a 2-stage parallel gearbox, and the high-speed shaft driving a three-phase induction generator.

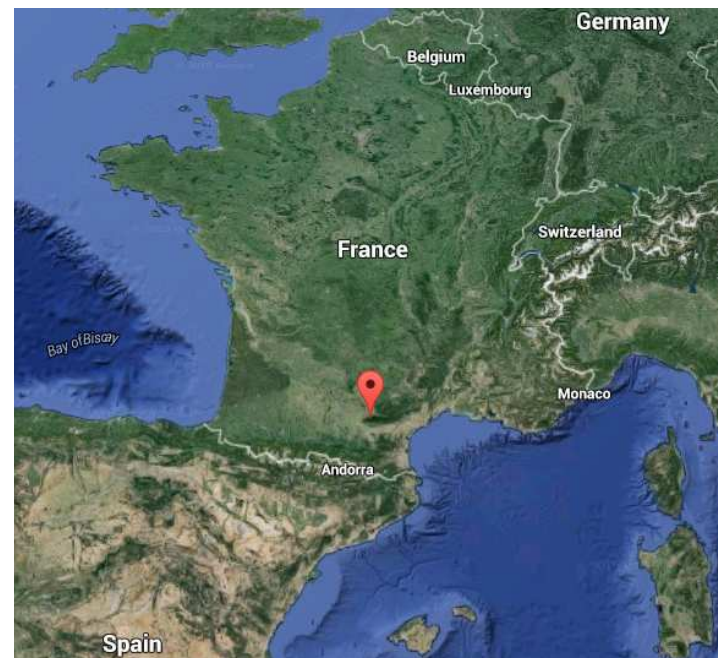

1.a location of the wind farm

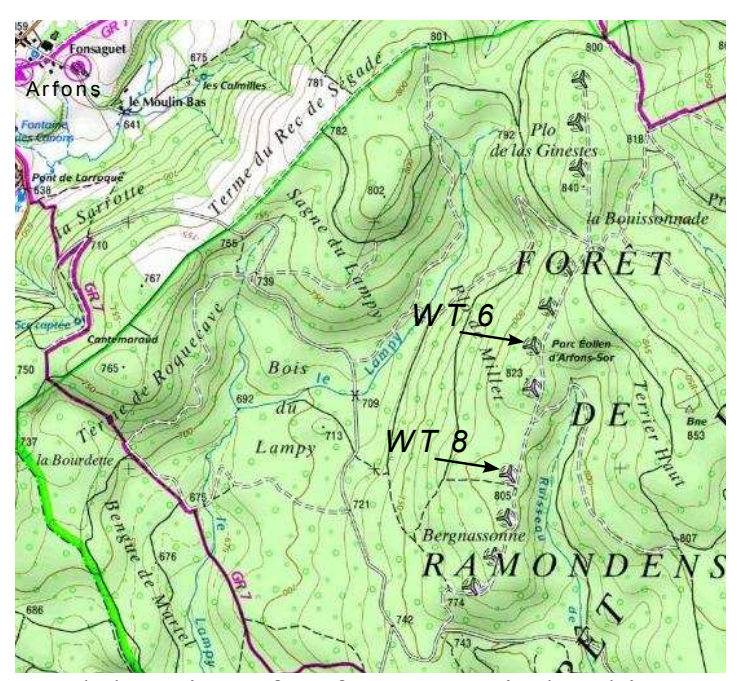

1.b location of Arfons-Sor wind turbines

Figure 1. Arfons-Sor wind farm [3]

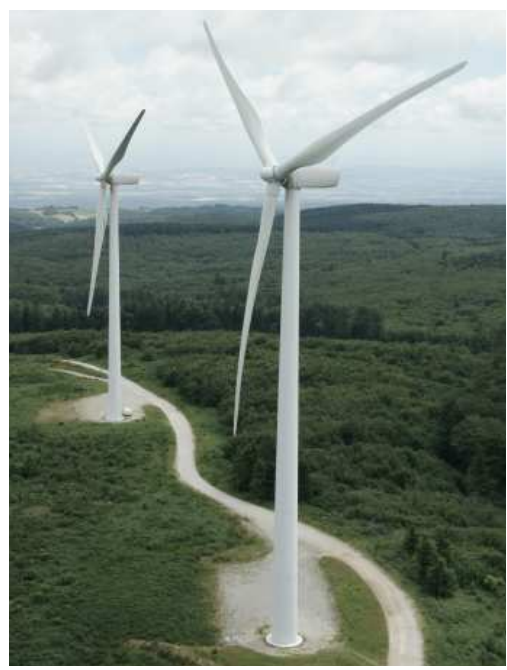

2.a Alstom Ecotecnia Eco 802.0

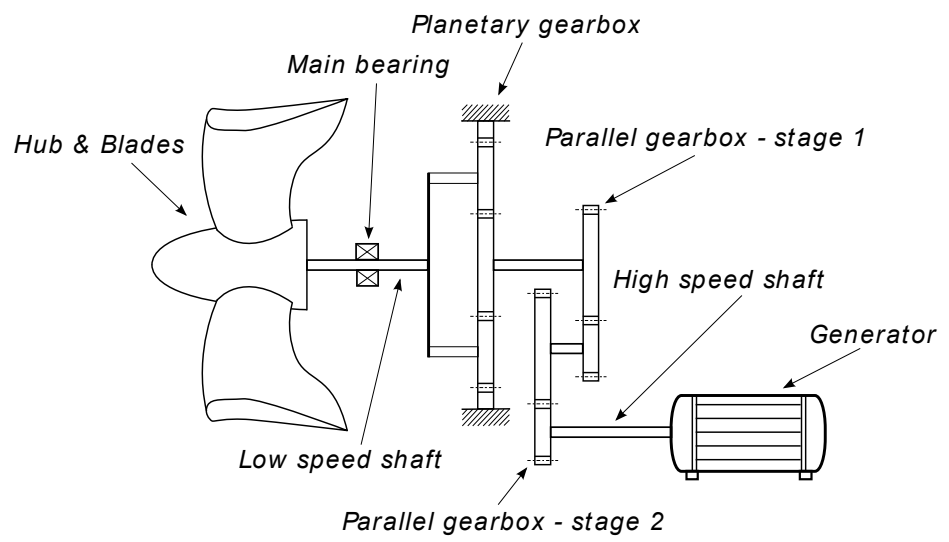

2.b main components of the drive train

Figure 2. Arfons-Sor wind turbines [3] 
In the context of the European project KAStrion presented in [1], two of these wind turbines (WT6 and WT8 in Fig. 1.b) were instrumented at the end of 2014 with an acquisition system and several mechanical and electrical sensors. Six accelerometers were mounted on different bearings of the drive train. A phase marker sensor was installed on the high-speed shaft for order-domain analysis and eventual angular resampling. Concerning electrical quantities, three current sensors and three voltage sensors were mounted on the stator of the electrical generator.

This set of sensors gives access to different physical quantities which were regularly acquired (at least once a day) for monitoring purpose during the beginning of 2015 with the following acquisition parameters:

- $\quad$ sampling frequency $=25 \mathrm{kHz}$,

- length of vibration signals $=40 \mathrm{~s}$,

- length of electrical signals $=10 \mathrm{~s}$.

In what follows, only one part of this large database is used to compare electrical and vibration analysis methods for mechanical faults monitoring:

- one vibration signal measured at the input bearing of the planetary gearbox,

- the three phase currents and line voltages of the stator of the electrical generator,

- the phase marker signal.

\section{Fault signature}

On the $30^{\text {th }}$ of December 2015, a total breakdown of wind turbine WT8 occurred. After inspection, a major mechanical fault located in the main bearing was diagnosed. More precisely, it was evident that an inner race fault continuously developed during a long period of time to finally end in the bearing partial destruction and locking.

In order to compare the detection capabilities of approaches based on electrical or vibration signals applied to this kind of fault, the signature of this fault in vibrations and electrical quantities must first be described.

\subsection{Fault signature in vibration data}

Inner race bearing faults generate mechanical impacts with a frequency [2]:

$$
f_{\mathrm{BPFI}}=\frac{N}{2} f_{\mathrm{R}}\left(1+\frac{d_{\mathrm{B}}}{d_{\mathrm{P}}} \cos \beta\right),
$$

where $N$ is the number of rolling elements, $f_{\mathrm{R}}<f_{\mathrm{BPFI}}$ is the shaft rotating frequency, $d_{\mathrm{B}}$ and $d_{\mathrm{P}}$ the ball and pitch diameters, and $\beta$ the contact angle.

In a $1^{\text {st }}$ approximation [10], these impacts can be assumed purely periodic with a fundamental frequency $f_{\mathrm{BPFI}}$, and amplitude modulated at the shaft rotating frequency $f_{\mathrm{R}}$ by the load effect. This is not the case in more refined models [11,12] where the contact angle depends on the ball position, leading to mechanical impacts containing also a random part. In both cases however, their low-frequency content is mainly constituted by frequency peaks with frequencies harmonically related to $f_{\text {BPFI }}$ surrounded by sidebands spaced by $f_{\mathrm{R}}$. These impacts generate mechanical waves 
travelling through structures to the sensor where the vibration signal is finally measured. Even by taking into account the time variation of the transfer function between the impact location and the sensor, the low-frequency content of the vibration signal due to this particular fault can be approximated by a series of peaks of frequencies $k \times f_{\mathrm{BPFI}} \pm l \times f_{\mathrm{R}}$ where $k$ and $l$ are integers and $f_{\mathrm{BPFI}}>f_{\mathrm{R}}$. These set of peaks finally constitutes one part of the vibratory signature of inner race bearing faults. Table 1 gives the values of these characteristic frequencies for the main bearing of the monitored wind turbine. They are expressed in orders of the high-speed shaft rotating frequency.

\begin{tabular}{|l|l|c|}
\hline$f_{\mathrm{BPFI}}$ & 0.31605 & ball pass frequency of inner race \\
\hline$f_{\mathrm{R}}$ & 0.00995 & low-speed shaft rotating frequency \\
\hline
\end{tabular}

Table 1. Characteristic frequencies of the faulty main bearing expressed in orders of the high-speed shaft rotating frequency [3]

\subsection{Fault signature in electrical data}

The mechanical impacts previously described have no effect on electrical quantities measured at the output of the wind turbine generator. However, bearing faults also generate load torque oscillations on the rotating shaft where the faulty bearing is located, which is the low-speed shaft in the present case. These torque variations occur at the same rate as the mechanical impacts and have the same characteristics. Therefore, they can also be approximated in the low-frequency range as a series of peaks with frequencies $k \times f_{\mathrm{BPFI}} \pm l \times f_{\mathrm{R}}$ ( $k$ and $l$ integers). If these torque oscillations reach the high-speed shaft by travelling through the drive train, they will affect the electrical generator and consequently its electrical quantities. In [4,5], such torque oscillations have been shown to cause amplitude and/or phase modulations in electrical quantities and more particularly in stator currents of induction machines. One part of the electrical signature of an inner race bearing fault is therefore the presence of amplitude and/or phase modulations of frequencies $k \times f_{\mathrm{BPFI}} \pm l \times f_{\mathrm{R}}$ ( $k$ and $l$ integers) in the stator currents of the output generator of the monitored wind turbine.

\section{Data selection process}

As mentioned in section 2, electrical and vibration data were regularly (at least daily) acquired on the monitored wind turbine from January to May 2015, enabling the constitution of a large database. The first task to do is then to select a correct data subset from the whole database enabling to simply and correctly monitor the wind turbine.

In order to simplify as much as possible the analysis methods used to monitor the bearing fault, the first criterion is to select signals which are as stationary as possible. This requirement is more or less equivalent to select the signals acquired with a shaft rotating frequency as constant as possible. Therefore, the variations of the shaft rotating frequency during each acquisition constitutes a first interesting feature to select the data. In order to easily compare the results obtained for different signals, the data should be acquired when the monitored wind turbine runs around the same operating point. This requirement is more or less equivalent to select the data acquired for similar shaft 
rotating frequency and electrical power generated. Therefore, mean values of the shaft rotating frequency and of the electrical power computed over the acquisition time constitute other interesting features to select the data.

From this and noting that vibration and electrical data were not acquired at the same time, the two following data selection strategies have been developed.

\subsection{Vibration data selection}

From the previous discussion, the useful features to select vibration data are the mean value of the shaft rotating frequency (representing the wind turbine operating point) and its maximum variation (representing the stationarity of signals) computed over the acquisition time. Therefore, for each acquired vibration signal, the following quantities are determined:

- The time evolution of the high-speed shaft rotating frequency is estimated thanks to the phase marker signal. This sensor gives one phase reference per round of the high-speed shaft, such that one estimation $f_{H}[n]$ of its rotating frequency is realized for each round $n$.

- The mean value $\bar{f}_{H}$ of $f_{H}[n]$ is then estimated over the whole acquisition time.

- In order to measure the variations of $f_{H}\lfloor n\rfloor$, several quantities can be estimated. The relative standard deviation $\sigma_{H}$ (usual standard deviation of $f_{H}[n]$ normalized by $\bar{f}_{H}$ ) and the relative maximum deviation $\Delta_{H}$ (maximum deviation of $f_{H}[n]$ normalized by $\bar{f}_{H}$ ) are the two chosen features. These two quantities are slightly different, $\sigma_{H}$ representing the small fluctuations of $f_{H}\lfloor n\rfloor$, while $\Delta_{H}$ being more sensitive to linear or polynomial trends.

These three features are computed over a preselected set of 92 vibration signals verifying $\bar{f}_{H}>26.5 \mathrm{~Hz}$ and $\sigma_{H}<4 \%$, and the obtained distributions are given in Fig. 3.a.
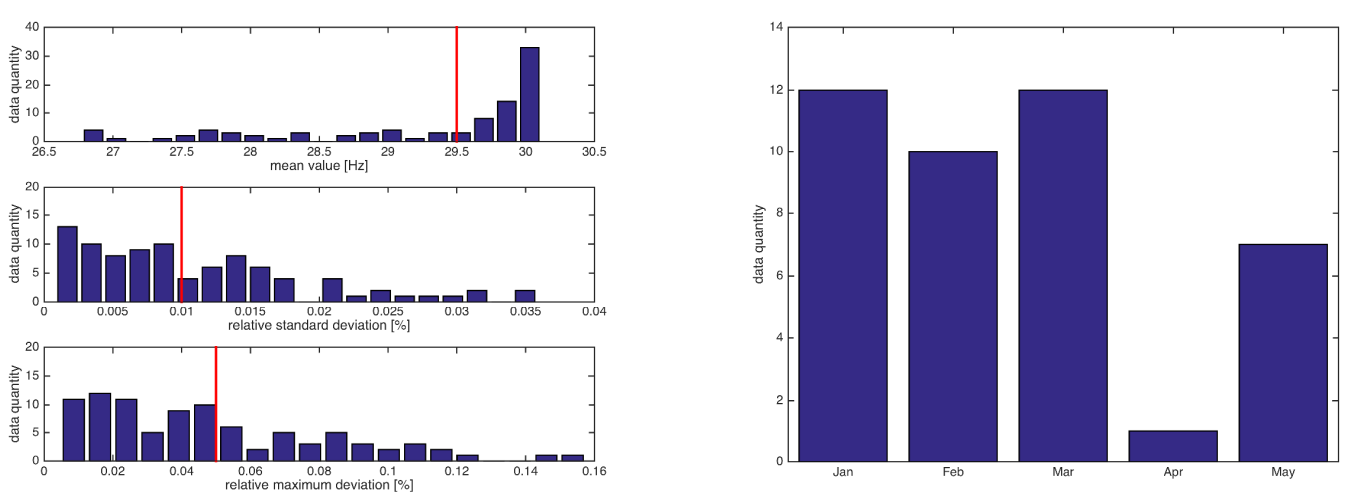

3.a distribution of shaft rotating frequency features (top $\bar{f}_{H}$, middle $\sigma_{H}$, down $\Delta_{H}$ )

3.b time distribution of selected data

Figure 3. Vibration data selection 
The values of $\bar{f}_{H}$ are clearly concentrated close to $30 \mathrm{~Hz}$, which is the nominal value of the electrical generator rotating frequency. The distributions of $\sigma_{H}$ and $\Delta_{H}$ show that the major part of their values stay close to 0 , whatever the acquired signal. Finally, data verifying $\bar{f}_{H}>29.5 \mathrm{~Hz}$ (the wind turbine works close to its nominal operating point) and $\sigma_{H}<1 \%$ and $\Delta_{H}<5 \%$ (the rotating frequency is almost constant and the signals almost stationary) are selected in the final dataset. The corresponding thresholds appear as red lines in Fig. 3.a, and finally lead to a set of 42 selected vibration signals. The distribution of these selected signals over time given in Fig. 3.b shows that the obtained vibratory database correctly covers the period of time during which the wind turbine is monitored (from January to May).

\subsection{Electrical data selection}

The electrical data selection strategy is exactly the same as for vibration data, but an additional criterion is used on the electrical quantities generated by the wind turbine generator to further specify its operating point. The idea is to keep electrical data sufficiently close to their maximal and nominal value. This can be done for example by computing the RMS value of each acquired electrical data, and discarding those for which this value is too small compared to its maximum. The electric data are finally selected if they also verify the previous criteria concerning the shaft rotating frequency.

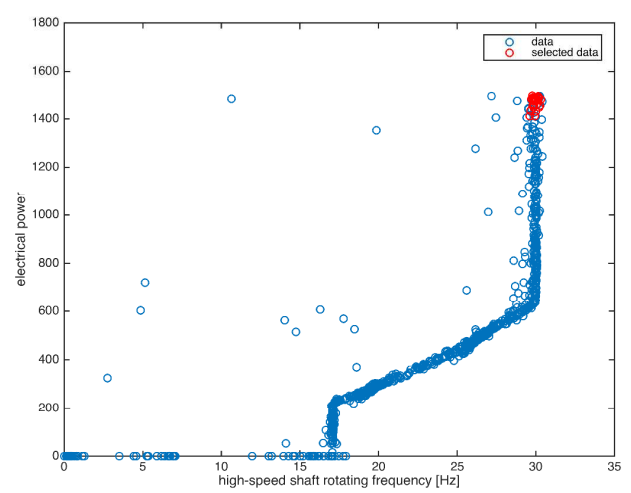

4.a data selection criteria

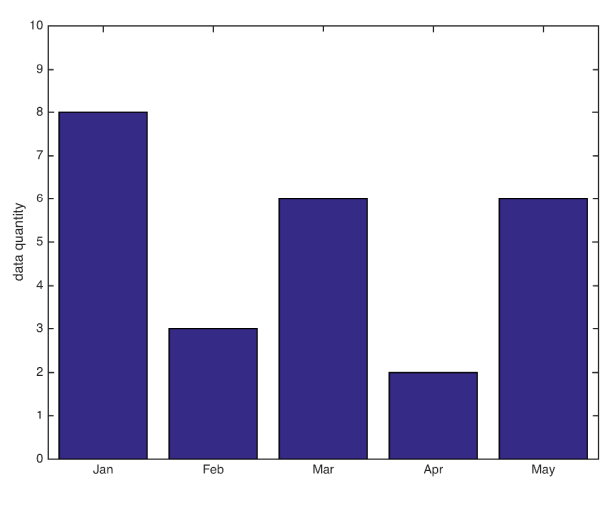

4.b time distribution of selected data

Figure 4. Electrical data selection

This selection strategy is applied to the whole electrical database consisting of 790 records, and leads to a set of only 25 electrical data. Fig. 4.a compares the whole electrical dataset (in blue) and the selected one (in red). In this figure, each data is represented as a point in a plane with $\bar{f}_{H}$ on the abscissa and the electric power (roughly estimated as the product of current and voltage RMS values) on the ordinate. Such a curve is quite similar to the power curve usually used to characterize the operating points of wind turbines. This figure clearly shows that every selected data was acquired when the wind turbine ran around the same operating point obtained for the nominal shaft rotating frequency and the nominal electrical power. Moreover, their time distribution represented in Fig. 4.b shows that the selected electrical database correctly covers the desired period of time going from January to May. 


\section{Vibration data results}

According to section 3.1, one part of the vibratory signature of an inner race bearing fault is constituted by a set of spectral peaks with frequencies $k \times f_{\mathrm{BPFI}} \pm l \times f_{\mathrm{R}}$ ( $k$ and $l$ integers), where $f_{\mathrm{BPFI}}$ is given by Eq. (1) and $f_{\mathrm{R}}<f_{\mathrm{BPFI}}$ is the low-speed shaft rotating frequency. The values of these frequencies expressed in orders of the high-speed shaft rotating frequency $f_{\mathrm{H}}$ are given in Table 1 .

The presence and the temporal evolution of this signature can be easily studied by computing the power spectral density of the selected vibration signals in the frequency band of interest. The chosen estimator is a simple averaged modified periodogram or Welch's periodogram [6], with a Blackman window of length 2.6s (corresponding to a spectral resolution of 0.02 orders of $f_{\mathrm{H}}$ ), an overlap of $75 \%$ and a zero-padding factor of 4. The obtained results are given in Fig. 6 where the PSDs obtained at the beginning of the period of interest (January) appear in blue and the ones obtained at the end (May) appear in red.
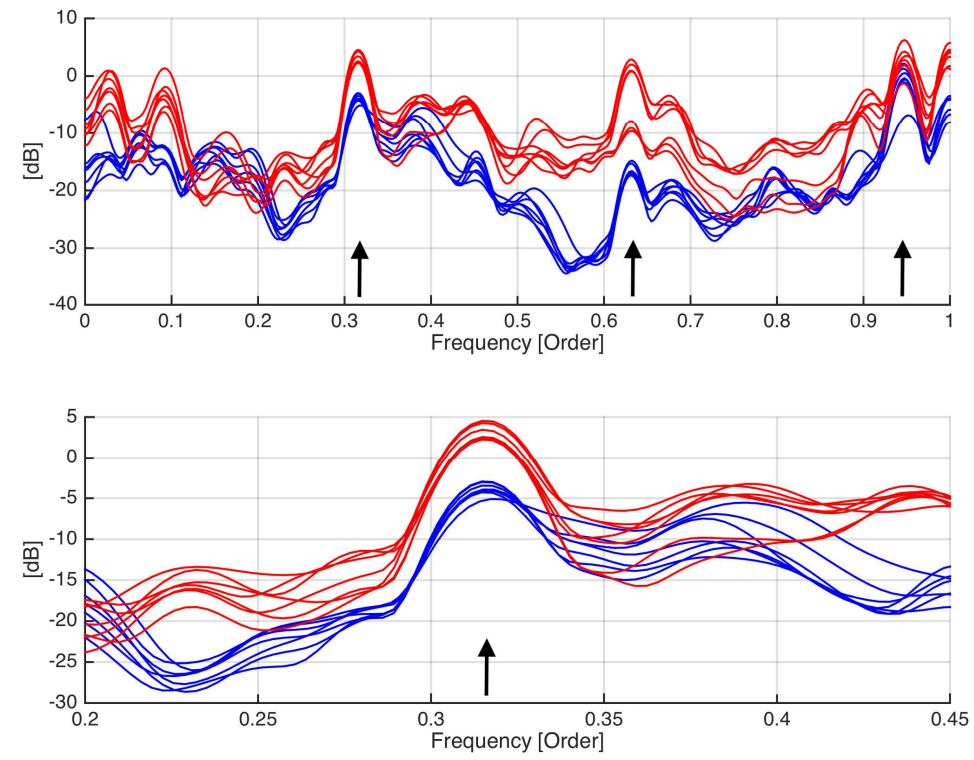

Figure 5. Power spectral densities of vibration signals - frequency in orders of $f_{\mathrm{H}}$

$$
\text { (blue }=\text { January, red }=\text { May) }
$$

The top figure shows a frequency band were the first 3 harmonics of $f_{\mathrm{BPFI}}$ labelled by black arrows are visible, contrarily to their sidebands hidden by a strong wide band noise and the limited spectral resolution. Clearly, the fault signature is already present in the first PSDs, but not with a significant level. Its amplitude is much more important in the last PSDs, highlighting a large increase in the fault signature between January and May. The down figure focuses on the $1^{\text {st }}$ harmonic of $f_{\mathrm{BPFI}}$, which is further used to compute a simple fault indicator obtained by summing the estimated PSDs around this particular frequency (more precisely between 0.29 to 0.34 ). 
Fig. 6 shows the temporal evolution of this indicator during the whole period of interest according to the measurement number (Fig. 6.a) and the measurement date (Fig. 6.b). A large increase of this quantity between the end of March and the end of April is clearly visible in these figures. Therefore, this simple vibration fault indicator shows that the condition of the main bearing inner race strongly deteriorates during the period of interest, and particularly during the month of April.

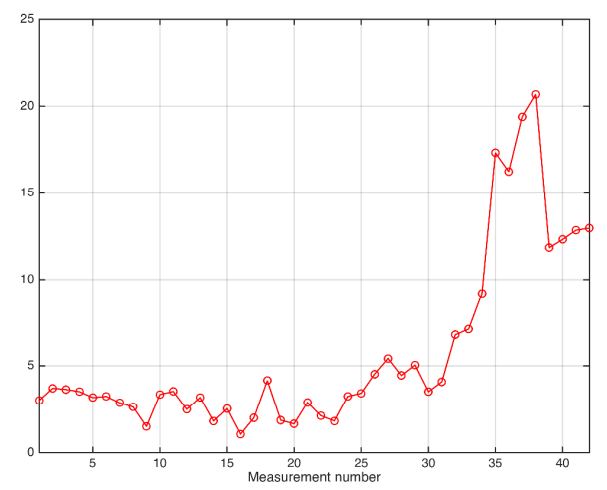

6.a evolution vs. measurement number

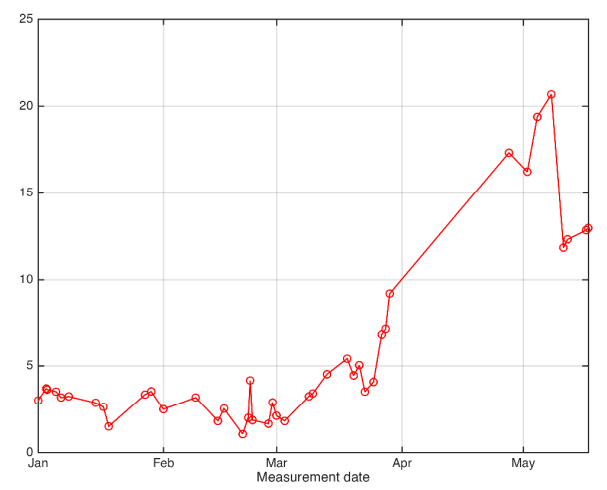

6.b evolution $v s$. measurement date Figure 6. Fault indicator obtained through vibration data

\section{Electrical data results}

According to section 3.2, one part of the electrical signature of the inner race bearing fault is the presence of amplitude and/or phase modulations of frequencies $k \times f_{\mathrm{BPFI}} \pm l \times f_{\mathrm{R}}$ ( $k$ and $l$ integers) in the stator currents measured at the output generator of the monitored wind turbine. A logical approach is then to demodulate the main component of these currents, in other words their fundamental component located at $f_{0}=50 \mathrm{~Hz}$, and check the presence of components of frequency $k \times f_{\mathrm{BPFI}} \pm l \times f_{\mathrm{R}}$ in the demodulated quantities.

A three-phase method was recently proposed in [7] to realize such a three-phase demodulation, jointly with a fault indicator dedicated to mechanical faults in threephase electrical rotating machines. The whole structure of this method is summarized in Fig. 7, where three main parts clearly appear.

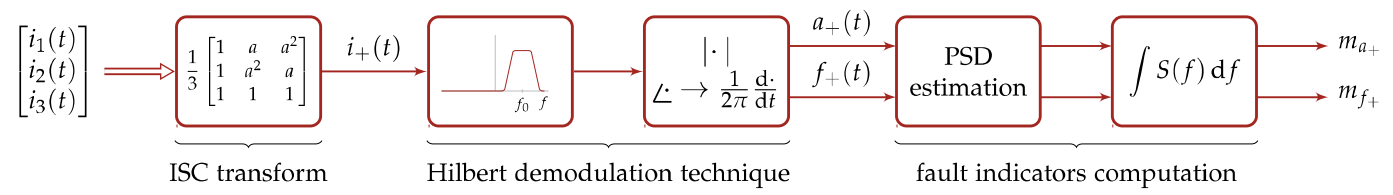

Figure 7. Mechanical fault indicator computation for three-phase electrical data

The first step of this algorithm is the application of the instantaneous symmetrical component (ISC) transform [8] to the three phase currents. It consists of a simple matrix product between the three measured stator currents and the inverse Fortescue matrix. This operation returns the three ISCs corresponding to the measured currents. The second step consists in applying a Hilbert demodulation technique [9] to the positive sequence ISC (noted $i_{+}(t)$ in Fig. 7) around the electrical fundamental frequency 
$f_{0}=50 \mathrm{~Hz}$. As explained in [7], the output of this second step is the instantaneous amplitude and frequency of the electrical fundamental component (noted $a_{+}(t)$ and $f_{+}(t)$ in Fig. 7). Once the three-phase quantities are demodulated, the third and last step is the fault indicator computation based on the integration of a simple power spectral density estimator. The two main advantages of using this three-phase approach compared to usual single-phase algorithms is that it uses the whole information contained in three-phase electrical quantities, and it improves the final signal-to-noise ratio in the demodulated quantities. As a direct consequence, the fault indicators proposed in Fig. 7 offer better detection capabilities than the ones based on single-phase approaches [7].

This approach is applied to the three-phase currents belonging to the selected electrical dataset described in section 4. Fig. 8 shows the spectral content of the demodulated electrical quantities, in other words of the instantaneaous amplitude $a_{+}(t)$ (Fig. 8.a top) and frequency $f_{+}(t)$ (Fig. 8.a - down) of the fundamental component located at $50 \mathrm{~Hz}$. These power spectral densities have been estimated with the same estimator as for vibration data. No clear modulating component increases in the spectrum of $a_{+}(t)$ at the expected frequency of $f_{\mathrm{BPFI}}$ labelled by black arrows. This shows that this quantity contains few information on such a bearing fault. On the contrary, this particular component increases in the spectrum of $f_{+}(t)$ during the period of interest, as highlighted by the focus of Fig. 8.b. Consequently, a simple fault indicator can be obtained by summing the power spectral density of $f_{+}(t)$ around the expected fault frequency $f_{\mathrm{BPF}}$ (and as for vibration data, between 0.29 and 0.34 ).
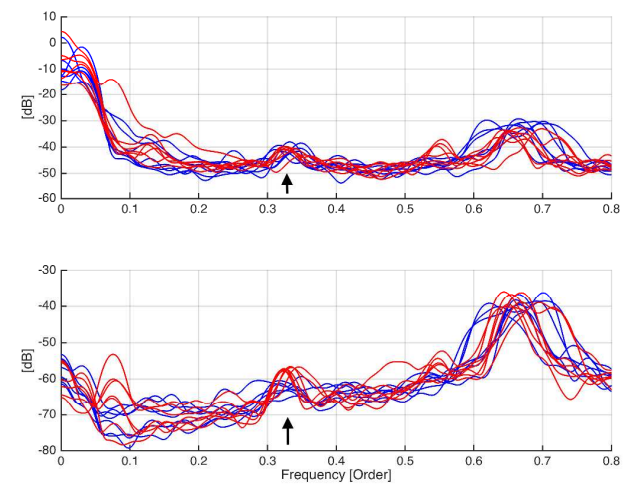

8.a instantaneous amplitude $a_{+}(t)$ (top) and frequency $f_{+}(t)$ (down)

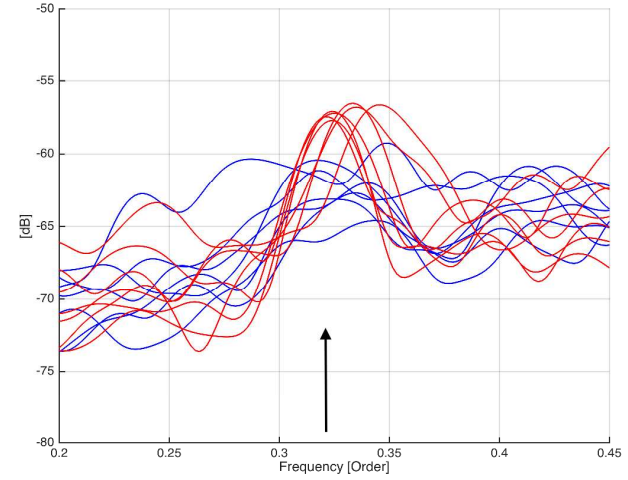

8.b instantaneous frequency $f_{+}(t)$

Figure 8. Power spectral densities of demodulated electrical quantities frequency in orders of $f_{\mathrm{H}}$ (blue = January, red = May)

Fig. 9 shows the temporal evolution of this electrical indicator during the whole period of interest according to the measurement number (Fig. 9.a) and the measurement date 
(Fig. 9.b). A slight increase of this quantity between the end of March and the end of April is visible in these two sub-figures. When comparing these results with the ones of Fig. 6, it is clear that the electrical indicator is less sensitive to the inner race fault than the mechanical indicator.

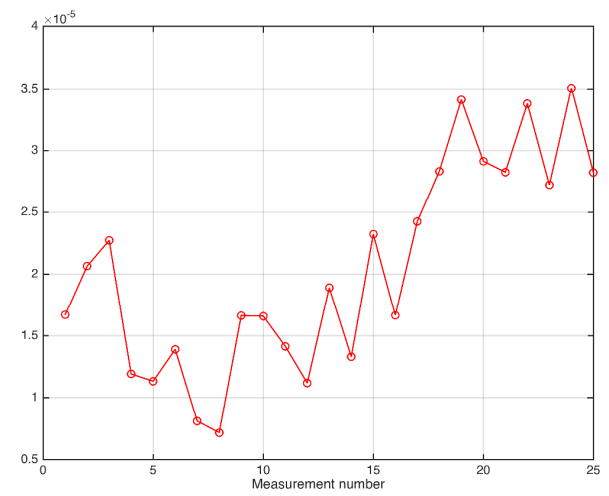

9.a evolution vs. measurement number

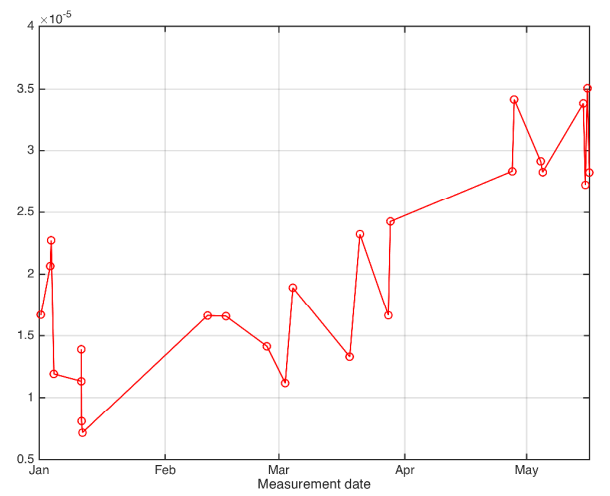

9.b evolution $v s$. measurement date

Figure 9. Fault indicator obtained through electrical data

However, An important point to notice is the complexity of the wind turbine drive train, composed of a planetary gearbox followed by a 2 -stage parallel gearbox. Indeed, the accelerometer delivering the vibration signal used to compute the mechanical indicator is located close to the main bearing and finally to the fault impacts. On the contrary, in order to influence the electrical generator, the load torque oscillations due to the main bearing fault must propagate through all the elements of the gearboxe to the high-speed shaft. As a consequence, their electrical signature in the stator currents is less important, and the fault detection is more difficult.

\section{Conclusions}

This paper compares electrical and vibratory condition monitoring methods to detect a mechanical fault occurring in the main bearing of an industrial wind turbine. Firstly, a data selection process uses the shaft rotating frequency and the electrical power at the output of the wind turbine in order to ensure that the processed data are stationary and acquired around the same operating point. Secondly, vibration and electrical data are processed on the same period of time to obtain two different mechanical fault indicators dedicated to inner race bearing faults. Clearly, these two indicators behave similarly and increase during the same month, indicating that an inner race fault probably developed in the wind turbine main bearing during this particular month. However, the indicator obtained through vibrations seems to be more sensitive than the one relying on electrical data. As mentioned in section 6, this is due to the fact that the fault is distant from the electrical generator located on the other side of the drive train. In such a case, the electrical indicator can be used to confirm the presence of a mechanical fault, but hardly to detect it with certainty. 


\section{References}

1. N. Martin, KAStrion project: a new concept for the condition monitoring of wind turbines, Twelfth International Conference on Condition Monitoring and Machinery Failure Prevention Technologies, CM 2015, Oxford, UK, 9-11 June 2015.

2. R. B. Randall and J. Antoni, Rolling element bearing diagnostics - a tutorial, Mechanical Systems and Signal Processing, Vol 25, pp. 485-520, 2011.

3. M. Firla, Automatic signal processing for wind turbine condition monitoring, $\mathrm{PhD}$. Univ. Grenoble Alpes, 2016.

4. M. Blödt, P. Granjon, B. Raison and G. Rostaing, Models for bearing damage detection in induction motors using stator current monitoring, IEEE Transactions on Industrial Electronics, Vol. 55, n. 4, pp. 1813-1822, 2008.

5. M. Blödt, P. Granjon, B. Raison and J. Régnier, Mechanical fault detection in induction motor drives through stator current monitoring - theory and application examples, Fault Detection, Wei Zhang (Ed.), 2010.

6. P. D. Welch, The use of the fast Fourier transform for the estiation of power spectra: a method based on time averaging over short modified periodograms, IEEE Transactions on Audio and Electroacoustics, Vol. 15, n. 2, pp. 70-73, June 1967.

7. G. Cablea, P. Granjon and C. Bérenguer, Three-phase electrical signals analysis for mechanical faults monitoring in rotating machine systems, Mechanical Systems and Signal Processing, Vol. 92, pp. 278-292, 2017.

8. G. C. Paap, Symmetrical components in the time domain and their application to power network calculations, IEEE Transaction on Power Systems, Vol. 15, n. 2, pp. 522-528, May 2000.

9. A. Reilly, G. Frazer and B. Boashash, Analytic signal generation - tips and traps, IEEE Transactions on Signal Processing, Vol 42, n. 11, pp. 3241-3245, 1994.

10. P. D. McFadden and J. D. Smith, Model for the vibration produced by a single point defect in a rolling element bearing, Journal of Sound and Vibration, Vol. 96, n. 1, pp. 69-82, 1984.

11. J. Antoni and R. B. Randall, The relationship between the spectral correlation and envelope analysis in the diagnostic of bearing faults and other cyclostationary machine signals, Mechanical Systems and Signal Processing, Vol. 15, n. 5, pp. 945962, 2001.

12. J. Antoni and R. B. Randall, A stochastic model for simulation and diagnostics of rolling element bearings with localized faults, Journal of Sound and Vibration, Vol. 125, pp. 282-289, July 2003. 